\title{
Influência do aumento do número de pupas hospedeiras de Cochliomyia macellaria (Diptera, Calliphoridae) no desenvolvimento do parasitóide Nasonia vitripennis (Hymenoptera, Pteromalidae) em laboratório
}

\author{
Leandro S. Barbosa ${ }^{1}$, Márcia S. Couri ${ }^{2,4} \&$ Valéria M. A. Coelho ${ }^{3}$
}

1. Programa de Pós-graduação em Ciências Biológicas (Zoologia), Museu Nacional, UFRJ. Bolsista CAPES. (leanbarbosa@globo.com)

2. Laboratório de Diptera, Departamento de Entomologia, Museu Nacional da Quinta da Boa Vista, 20940-040 Rio de Janeiro, RJ. (mcouri@terra.com.br)

3. Laboratório de Estudo de Dípteros, Departamento de Microbiologia e Parasitologia, UNIRIO, Rua Frei Caneca 94, 20211-040, Rio de Janeiro, RJ. (valeria@unirio.br)

4. Bolsista de Produtividade em Pesquisa, 1B, CNPq.

\begin{abstract}
Influence of the increase of the number of the host pupae of Cochliomyia macellaria (Diptera, Calliphoridae) in the development of the parasitoid Nasonia vitripennis (Hymenoptera, Pteromalidae) in laboratory. The post-embryonic development, productivity, rate of parasitism and sexual ratio of Nasonia vitripennis (Walker, 1836) reared in pupae of Cochliomyia macellaria (Fabricius, 1775) were studied. Different densities of the host were used (proportion 1:1, 1:2, 1:3, 1:4, and 1:5) and the exposition in each one lasted 72 hours. Nulliparous females originating from the stock colony were individualized in test tubes covered with hydrophobic cotton and containing the host pupae. Each treatment was constituted by 10 repetitions. After the exposition, the wasps were discarded and the host pupae were individualized in test tubes until the emergence of the adults of $C$. macellaria or $N$. vitripennis. Samples of host pupae not exposed to parasitism and receiving the same experimental treatment were used as control. The parasitoids showed a slower development in relation to the increase of host density. There was a production decrease of parasitoids per host in elevated densities. The sexual ratio tended towards a deviation for the birth of females with the increase of the density of host. The rate of parasitism showed a decrease when the parasitoid was exposed to more than two host pupae.
\end{abstract}

KEYWORDS. Biological control, wasp, fly, parasitism.

RESUMO. O desenvolvimento pós-embrionário, a produtividade, a taxa de parasitismo e a razão sexual de Nasonia vitripennis (Walker, 1836) criada em pupa de Cochliomyia macellaria (Fabricius, 1775) foram estudados. Densidades diferentes de hospedeiro foram usadas (proporções 1:1, 1:2, 1:3, 1:4 e 1:5) com tempo de exposição de 72 horas, em cada uma delas. Fêmeas nulíparas originárias da colônia estoque foram individualizadas em tubos de teste cobertos com algodão hidrófobo e contendo as pupas hospedeiras. Cada tratamento constituiu-se de 10 repetições. Depois da exposição, as vespas foram descartadas e as pupas hospedeiras foram individualizadas em tubos de teste até a emergência dos adultos de C. macellaria ou $N$. vitripennis. Amostras das pupas hospedeiras não expostas ao parasitismo e recebendo o mesmo tratamento experimental foram usadas como controle. Os parasitóides mostraram um desenvolvimento mais lento em relação ao aumento da densidade de hospedeiros. Houve uma diminuição na produção de parasitóides por hospedeiro em densidades elevadas. A razão sexual tendeu para um desvio para nascimento de fêmeas com o aumento da densidade do hospedeiro. Os índices de parasitismo mostraram uma diminuição quando o parasitóide foi exposto a mais de duas pupas hospedeiras.

PALAVRAS-CHAVE. Controle biológico, vespas, moscas, parasitismo.

Cochliomyia macellaria (Fabricius, 1775) (Diptera, Calliphoridae) é a principal espécie causadora de míase secundária no Brasil. Devido ao hábito necrófago das suas larvas, este díptero geralmente se encontra associado a outras espécies de califorídeos, sarcofagídeos e muscídeos. Veicula mecanicamente, através da superfície das pernas, corpo e conteúdo digestivo, diversos agentes patogênicos, apresentando importância médica, veterinária e econômica (BAUMGARTNER \& GREENBERG, 1985). No Brasil, assume relevância maior em virtude de ser uma das espécies veiculadoras de ovos de Dermatobia hominis (Linnaeus, 1781) (Diptera, Oestridae), mosca responsável pela miíase furuncular ou dermatobiose que causa muitos prejuízos à pecuária nacional (GUIMARÃES et al., 1983; MOYA-BORJA, 2003). Sua distribuição está limitada às Américas, ocorrendo tanto na região Neotropical como na Neártica, desde a Patagônia até o sul do Canadá incluindo as ilhas Galápagos e Antilhas (FERREIRA, 1983; BAUMGARTNER \& GREENBERG, 1984 e 1985).
Nasonia vitripennis (Walker, 1836) (Hymenoptera, Pteromalidae) encontra-se amplamente difundida pelo mundo, sendo considerada cosmopolita (RUEDA \& AxTELL, 1985). Devido ao comportamento gregário, esta espécie aponta vantagens para ser usada em programas de controle biológico aplicado de dípteros muscóides, tendo em vista a facilidade de criação em laboratório.

A taxa de reprodução de $N$. vitripennis pode ser potencializada com a utilização de hospedeiros de maior porte, utilizando como hospedeiros preferenciais representantes das famílias Calliphoridae e Sarcophagidae (CARDoso \& Milward-DE-AzEvedo, 1995).

WHITING (1967) apontou em sua revisão 68 espécies de hospedeiros de $N$. vitripennis e atualmente vem sendo descobertas novas espécies hospedeiras (MARCHIORI et $a l ., 2001)$. No presente trabalho, C. macellaria é apontada, pela primeira vez, como hospedeiro de $N$. vitripennis.

Este trabalho teve como objetivos avaliar o 
desenvolvimento pós-embrionário, a quantidade da prole de parasitóides por hospedeiro, a razão sexual da mesma e a taxa de parasitismo de $N$. vitripennis criadas em pupas de $C$. macellaria em diferentes densidades do hospedeiro. Visa também contribuir para um maior conhecimento na área de criação de $N$. vitripennis, fomentando assim, ações de controle biológico de dípteros muscóides.

\section{MATERIAL E MÉTODOS}

Criação dos insetos. O estabelecimento da colônia de C. macellaria foi realizado através da captura de, aproximadamente, 400 indivíduos adultos em área rural, na cidade de Paracambi, Rio de Janeiro. As coletas foram realizadas com auxílio de armadilha típica para coleta de lepidópteros, na qual foi adaptada uma manga para facilitar a captura dos dípteros vivos. Como isca utilizouse sardinha com 24 horas de exposição ao ambiente. Para a identificação dos exemplares utilizou-se a chave taxonômica de Mello (2003).

A colônia estoque dos dípteros foi mantida de acordo com a metodologia estabelecida por CUNHA-E-SILVA \& Milward-De-Azevedo (1994); Paes \& Milward-DeAzEvedo (1998), sendo os adultos criados em gaiolas teladas de aproximadamente $45 \mathrm{~cm}^{3}$, em temperatura ambiente. Estes receberam como fonte de alimento, solução de mel e água a 50\% e água ad libitum. Leite em pó e carne de origem bovina foram introduzidos do $1^{\circ}$ até o $5^{\circ}$ dia após a emergência, para a maturação dos ovários e, a partir do $10^{\circ}$ dia, a carne foi reintroduzida como substrato de oviposição. Os ovos, na relação aproximada de um ovo/grama de dieta, foram transferidos para recipiente plástico retangular de $400 \mathrm{ml}$ de capacidade, contendo carne bovina. Este, por sua vez, foi acondicionado em outro recipiente de dois litros, contendo maravalha, que serviu como substrato de pupariação, o qual foi vedado com tecido de náilon, preso com elástico e mantido com uma capela de criação de larvas com sistema de exaustão de gases.

A carne bovina utilizada foi obtida em açougue, cortada em cubos de aproximadamente $2 \mathrm{~cm}^{3}$, acondicionada em saco plástico em quantidade padronizada (aproximadamente $300 \mathrm{~g}$ ) e mantidas congeladas em refrigerador. $\mathrm{O}$ descongelamento da carne foi realizado na geladeira $\left(12^{\circ} \mathrm{C}\right)$ de forma gradual, 48 horas antes de ser oferecida aos adultos.

Visando a manutenção da colônia estoque de $N$. vitripennis, pupas de C. macellaria, com, no máximo 24 horas, foram acondicionadas em sacos plásticos, em lotes de aproximadamente 150 unidades e congeladas. Cada lote foi identificado com a data de congelamento.

A colônia estoque de $N$. vitripennis foi estabelecida a partir de coletas realizadas no Jardim Zoológico do Rio de Janeiro (Fundação Rio-Zoo). Para captura dos parasitóides foi utilizada uma gaiola de, aproximadamente $45 \mathrm{~cm}^{3}$, própria para criação de moscas, cuja tela permitia a entrada dos parasitóides, porém evitava a predação das pupas por outros animais. No interior das mesmas, como iscas, foram expostas pupas de Chrysomya megacephala (Fabricius, 1794) (Diptera: Calliphoridae) com aproximadamente 24 horas de idade, provenientes de colônia estoque, e carne bovina putrefata como fonte de apneumônio. Dois dias após a exposição no campo, as pupas foram recolhidas e acondicionadas em frascos de vidro transparentes $(13 \mathrm{~cm}$ de diâmetro x $24 \mathrm{~cm}$ de altura) vedados com tecido de algodão, até a emergência dos parasitóides.

A identificação dos parasitóides recém-emergidos foi realizada por meio da descrição taxonômica detalhada de RUEDA \& AXTELL (1985), e confirmada pela especialista no grupo Dra. Maria Angélica Penteado Dias (Departamento de Ecologia e Biologia Evolutiva da Universidade de São Carlos, São Paulo).

A colônia estoque dos microhimenópteros foi mantida em câmara climatizada $\left(25 \pm 1^{\circ} \mathrm{C}, 60 \pm 10 \%\right.$ U.R., fotofase de 14 horas), de acordo com metodologia recomendada por CARDoso \& MILwARD-DE-AzEvEDO (1996). Os adultos foram mantidos em gaiolas confeccionadas com recipiente plástico translúcido de um litro, recebendo, como alimento, uma solução de mel e água (1:1) embebida em algodão, dentro de um recipiente plástico com $4 \mathrm{~cm}$ de diâmetro.

Pupas de C. macellaria previamente congeladas por no máximo dois meses, foram depositadas em papel de filtro e descongeladas, com auxílio de uma luminária incidindo sobre as mesmas por 10 minutos. Estas foram distribuídas em placas de Petri teladas com filó e expostas aos parasitóides recém emergidos, possibilitando a retirada dos parasitóides do frasco por peneiração ao final do período de exposição. A exposição ocorreu por períodos de 48 horas. Após a exposição, as pupas foram alocadas em recipientes cilíndricos telados com tecido de náilon (escaline), identificados com a geração, data, período de exposição e provável data de emergência, e mantidos em câmara climatizada regulada com mesmas condições da colônia estoque.

Experimentos. A etapa experimental foi conduzida em câmara climatizada (Quimis) $\left(27 \pm 1^{\circ} \mathrm{C}, 60 \pm 10\right.$ U.R., fotofase 14 horas). A fotofase foi iniciada às $6 \mathrm{~h}$ da manhã.

Para testar os parâmetros biológicos, foram utilizadas fêmeas nulíparas de $N$. vitripennis pertencentes a $23^{\mathrm{a}}$ geração, com até 24 horas de idade, medindo de 2 a $3 \mathrm{~mm}$, previamente alimentadas com solução de água e mel (1:1). Utilizaram-se como hospedeiros, pupas frescas de $C$. macellaria, oriundas da $19^{\mathrm{a}}$ geração, com até 24 horas de idade e com peso corporal registrado entre 48 e $49 \mathrm{mg}$.

Fêmeas nulíparas do parasitóide, provenientes da colônia estoque, foram individualizadas em tubos de ensaio com $50 \mathrm{ml}$ de capacidade e tampados com algodão hidrófobo, contendo uma, duas, três, quatro ou cinco pupas hospedeiras. Cada tratamento foi constituído por 10 repetições. No interior de cada tubo inseriu-se uma tira de papel filtro embebido em solução de água e mel (2:1). Após 72 horas de exposição, as vespas foram descartadas e as pupas hospedeiras individualizadas em tubos de ensaio, onde se aguardou a emergência de $C$. macellaria ou $N$. vitripennis.

Lotes de pupas hospedeiras não expostas ao parasitismo, mantidas sob o mesmo delineamento experimental, foram utilizados como controle para verificar a taxa de mortalidade natural. As observações foram feitas diariamente, sob microscópio estereoscópico sempre no mesmo horário, até o $35^{\circ}$ dia após a exposição. 
Analisaram-se dados referentes à duração do desenvolvimento pós-embrionário, o número de parasitóides emergidos por pupa hospedeira, razão sexual e taxa de parasitismo. Considera-se a taxa de parasitismo o número de pupas de $C$. macellaria que originaram parasitóides.

Para testar a significância dos resultados foram utilizados a Análise de Variância e o Teste de Tukey através do programa GraphPad Prism 4.03 (MAGALHÃes \& LiMA, 2001).

\section{RESULTADOS}

A duração média do desenvolvimento pósembrionário de machos e fêmeas de $N$. vitripennis variou significativamente entre os tratamentos com diferentes densidades de pupas hospedeiras pela análise de variância $(\mathrm{P}<0,001 ; \mathrm{F}=6,16)$. O maior tempo de desenvolvimento verificado foi na relação 1:5 (14,66 dias), considerando toda a prole. Foi observado, para machos, um menor tempo de desenvolvimento pós-embrionário na relação $1: 1$, e um aumento do tempo nas densidades 1:2 e 1:3, e esta última, não diferindo significativamente das demais. Já para as fêmeas não foi verificada diferença significativa entre as densidades parasitóide:hospedeiro (Tab. I).

O pico de emergência de $N$. vitripennis ocorreu, nas densidades 1:1, 1:2 e 1:3, no $14^{\circ}$ dia após a exposição do parasitóide ao hospedeiro (Fig. 1). Na densidade de 1:4 observou-se um pico de emergência no $15^{\circ}$ dia. Na relação 1:5, apesar de observar-se um pico no $14^{\circ}$ dia, ocorreu um segundo pico com menor intensidade no $16^{\circ}$ dia.

Observou-se uma redução significativa da produção de machos nas densidades mais elevadas. $\mathrm{O}$ mesmo não foi verificado para as fêmeas (Tab. II). O número médio de parasitóides emergidos por pupa hospedeira variou significativamente entre as diferentes densidades de parasitóide: hospedeiro (ANOVA, $\mathrm{F}=5,28$; $\mathrm{p}=0,0006)$. O teste Tukey mostrou diferença significativa nas relações 1:1 e 1:2, que originaram maior número de parasitóides por pupa, em comparação com as demais densidades de 1:3, 1:4 e 1:5 (Tab. II).

O gráfico de tendência exponencial (Fig. 2) confirma a tendência ao nascimento de um maior número de fêmeas em função do aumento da densidade de pupas, alterando a razão sexual da prole de acordo com a disponibilidade de hospedeiros.
Observou-se uma redução na taxa de parasitismo de $C$. macellaria por $N$. vitripennis com o aumento do número de hospedeiros nas relações acima de 1:2, declinando até $86 \%$ na relação 1:5 (Fig. 3). Observaramse pupas inviáveis nas densidades 1:3 (12\%), 1:4 (5\%) e em 1:5 (8\%). Nesta última densidade, observou-se também a emergência de dípteros $(6 \%)$, confrontando com os controles que apresentaram nas relações de 1:1, 1:2 e 1:3 uma taxa de $100 \%$ de emergência de dípteros. Porém, os controles das relações 1:4 e 1:5 apresentaram índices reduzidos (2\%) de pupas inviáveis (Fig. 3).

\section{DISCUSSÃO}

Os resultados relativos ao desenvolvimento pósembrionário de $N$. vitripennis criadas em pupas de $C$. macellaria, utilizando-se diferentes densidades do hospedeiro, mostraram uma tendência à extensão da duração do desenvolvimento pós-embrionário em função do aumento do número de hospedeiros. Esta tendência foi também observada por CARDOSO \& MILWARD-DEAzEvedo (1995) em estudo sobre a influência do aumento da densidade de $C$. megacephala sobre o desenvolvimento de $N$. vitripennis, onde na densidade $1: 1$, as fêmeas e os machos apresentaram média de desenvolvimento 13,21 e 13,34 dias (24 horas de exposição), e 13,22 e 13,39 dias (48 horas de exposição) respectivamente, atingindo na densidade 1:5 valores de 13,41 e 13,39 dias (24 horas) e 13,74 e 13,89 dias (48 horas), respectivamente.

É sabido que a duração do desenvolvimento pósembrionário é diretamente influenciada pelas condições ambientais. E, no caso de insetos parasitóides, o desenvolvimento também é afetado pelo tamanho, bem como a espécie do hospedeiro (CARDOSO \& MILWARD-DEAzEvedo, 1996; HARvey \& Gols, 1998). Observou-se no presente estudo, à temperatura de $27^{\circ} \mathrm{C}$ e umidade relativa de $60 \pm 10 \%$, uma duração de desenvolvimento que variou de 14,28 a 14,66 dias após a exposição, porém SCHIMIDT (1986), à mesma temperatura, observou uma duração de 13 dias utilizando como hospedeiro Chrysomya rufifacies (Macquart, 1843), cujo pupário é maior. CARDOSO \& Milward-De-Azevedo (1996) observaram que o fator tamanho e espécie também afetam a quantidade de parasitóides que emergem por hospedeiro, relatando que pupas de Chrysomya albiceps (Wiedemann, 1819) oriundas de larvas mais robustas que as de $C$.

Tabela I. Duração média do desenvolvimento pós-embrionário (em dias) de Nasonia vitripennis criadas em pupas de Cochliomyia macellaria com até $24 \mathrm{~h}$, expostas ao parasitismo por fêmeas nulíparas individualizadas $(\mathrm{n}=10 /$ tratamento) com até $24 \mathrm{~h}$ pós-emergência, por por 72 horas, utilizando-se diferentes relações parasitóide/hospedeiro ${ }^{2}\left(\mathrm{~T}: 27^{\circ} \mathrm{C}, 60 \%\right.$ U.R., fotofase 14 horas). (X, média; s, desvio padrão; IV, intervalo de variação). Médias seguidas pela mesma letra não diferem significativamente pelo teste Tuckey em nível de 5\% de significância.

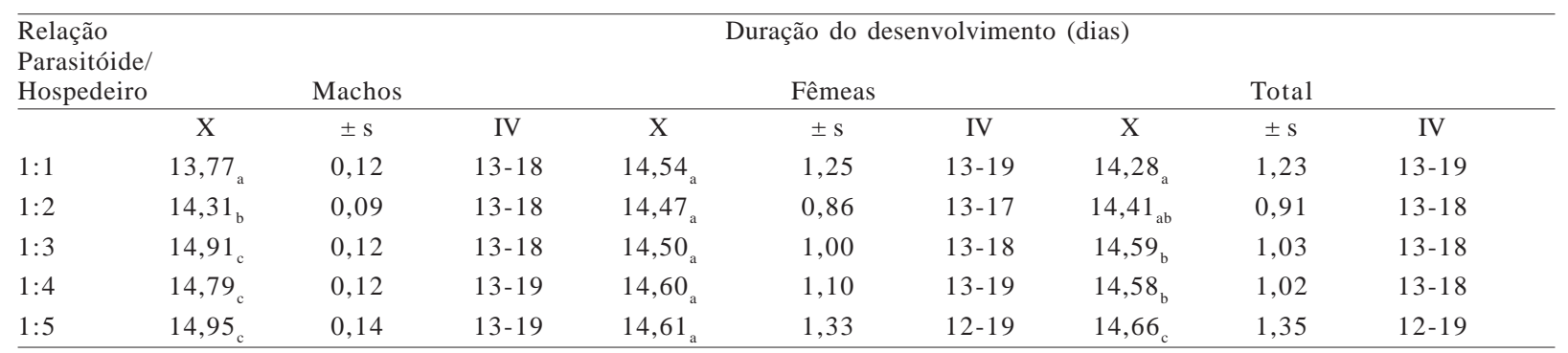


1 Parasitóide: 1 Hospedeiro

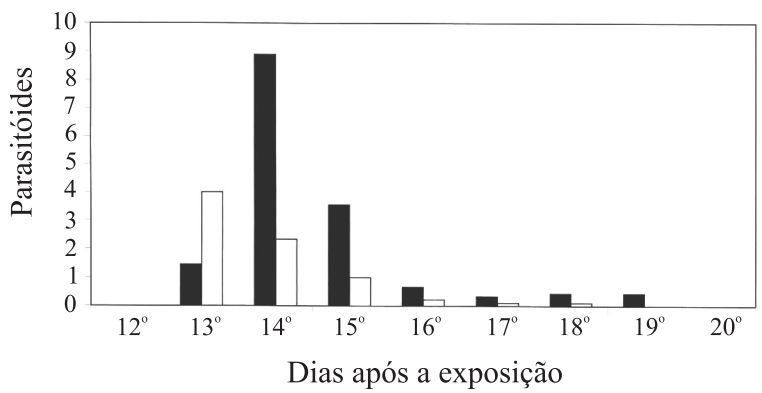

1 Parasitóide: 3 Hospedeiros

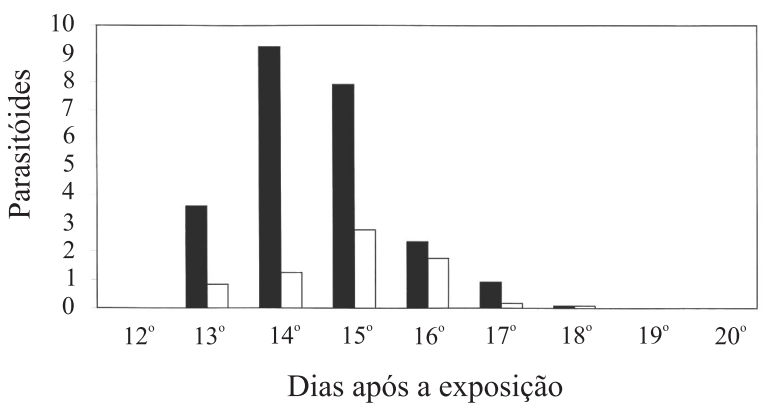

1 Parasitóide: 5 Hospedeiros

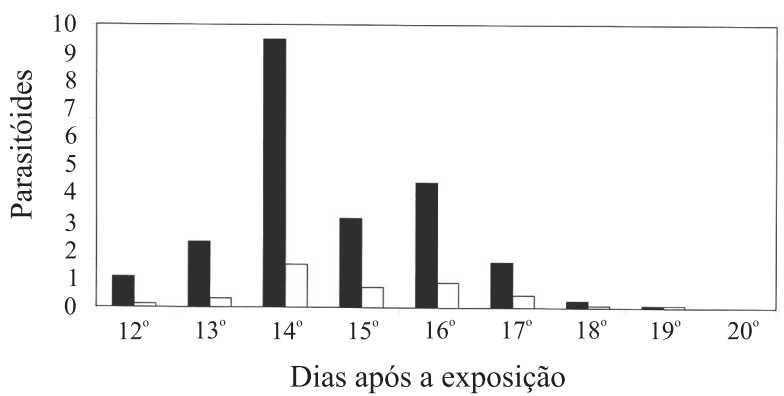

1 Parasitóide: 2 Hospedeiros

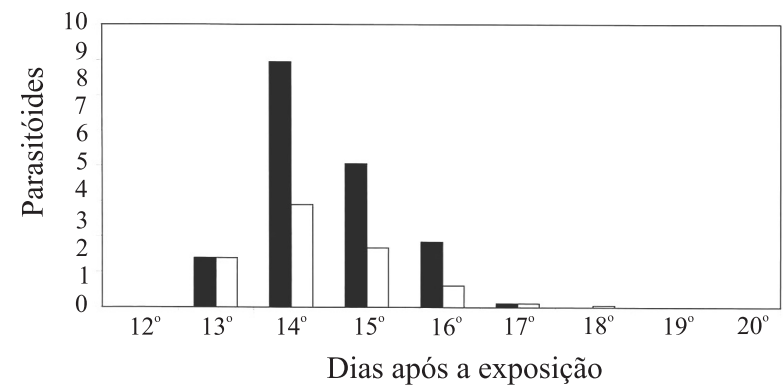

1 Parasitóide: 4 Hospedeiros

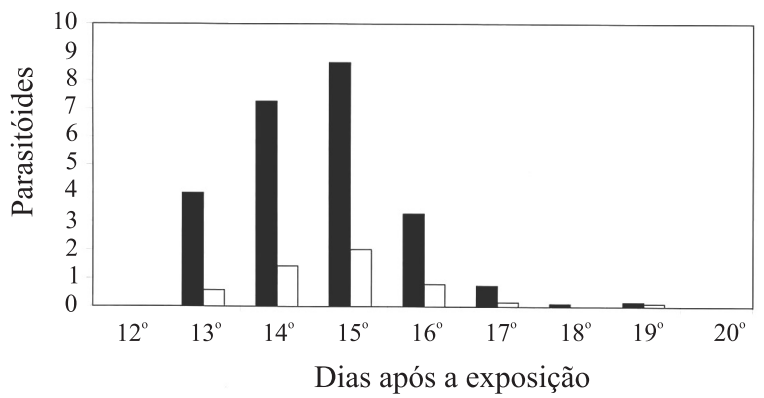

Fig. 1. Ritmo de emergência de fêmeas (preto) e machos (branco) de Nasonia vitripennis criadas em pupas de Cochliomyia macellaria expostas por 72 horas a fêmeas nulíparas, utilizando-se diferentes relações fêmeas parasitóides/hospedeiro.

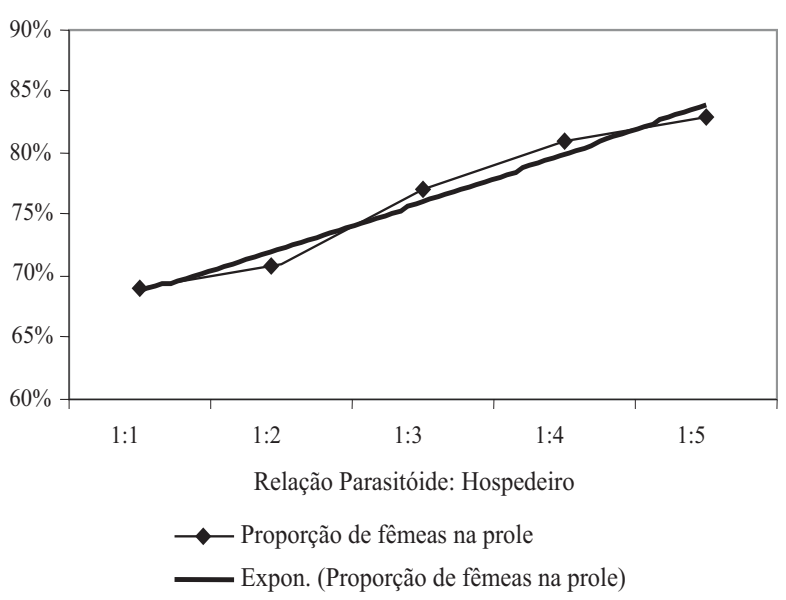

Fig. 2. Tendência exponencial da taxa de fêmeas na prole de Nasonia vitripennis criadas em pupas de Cochliomyia macellaria expostas por 72 horas a fêmeas nulíparas, utilizando-se diferentes relações fêmeas parasitóides/hospedeiro. megacephala permitiram a formação de um maior número de parasitóides. Utilizando hospedeiros com peso médio de $72 \mathrm{mg}$ (C. megacephala) e $77 \mathrm{mg}$ (C. albiceps) obtiveram em média a emergência de 46 e 49 insetos, respectivamente.

Os picos de emergência dos parasitóides, no presente estudo, tenderam a ser mais tardios com o aumento da densidade de hospedeiros. Gulias-Gomes et al. (2003) trabalhando com C. megacephala, a $27^{\circ} \mathrm{C}$ e umidade relativa de UR $65 \pm 10 \%$ observaram picos de emergência concentrados entre o $14^{\circ}$ e $15^{\circ}$ dia após o início da exposição, na relação 1 parasitóide: 2 hospedeiros, corroborando os dados do presente estudo. Os picos de emergência para machos e para fêmeas, no presente trabalho, obedeceram à mesma tendência, diferindo apenas na relação 1:1 em que foi observado um pico de emergência de machos precedente ao das fêmeas. Durações de desenvolvimento similares entre machos e fêmeas também foram constatadas por CARDOSO \& MiLWARD-DE-AZEVEDo (1995). 
Tabela II. Razão sexual e número de parasitóides adultos de Nasonia vitripennis criados por pupa de Cochliomyia macellaria exposta ao parasitismo por 72 horas, utilizando-se diferentes relações parasitóide/hospedeiro ${ }^{2}$ (T: $27^{\circ} \mathrm{C}, 60 \%$ U.R., Fotofase 14 horas). . (Razão sexual, média de fêmeas /Total; X, média; IV, intervalo de variação). Médias seguidas pela mesma letra não diferem significativamente pelo teste Tukey em nível de 5\% de significância.

\begin{tabular}{|c|c|c|c|c|c|c|c|}
\hline \multirow{3}{*}{$\begin{array}{l}\text { Relação } \\
\text { Parasitóid } \\
\text { Hospedeir }\end{array}$} & \multirow{3}{*}{$\begin{array}{l}\text { Razão } \\
\text { /sexual }\end{array}$} & \multicolumn{6}{|c|}{$\mathrm{N}^{\circ}$. de parasitóides adultos } \\
\hline & & \multicolumn{2}{|c|}{ Machos } & \multicolumn{2}{|c|}{ Fêmeas } & \multicolumn{2}{|c|}{ Total } \\
\hline & & $\mathrm{X}$ & IV & $\mathrm{X}$ & IV & $\mathrm{X}$ & IV \\
\hline $1: 1$ & 0,69 & $7,77_{\mathrm{a}}$ & $2-30$ & $17,75_{\mathrm{a}}$ & $13-24$ & $23,56_{a}$ & $16-30$ \\
\hline $1: 2$ & 0,71 & $6,72_{\mathrm{a}}$ & $1-31$ & $16,13_{\mathrm{a}}$ & $10-23$ & $21,06_{\mathrm{a}}$ & $14-31$ \\
\hline $1: 3$ & 0,77 & $3,45_{\mathrm{ab}}$ & $1-13$ & $12,04_{b}$ & $3-23$ & $15,46_{\mathrm{b}}$ & $5-26$ \\
\hline $1: 4$ & 0,81 & $3,00_{\mathrm{b}}$ & $1-8$ & $13,16_{\mathrm{ab}}$ & $4-20$ & $15,16_{\mathrm{b}}$ & $2-22$ \\
\hline $1: 5$ & 0,83 & $2,74_{b}$ & $1-7$ & $13,38_{\mathrm{ab}}$ & $3-24$ & $15,86_{b}$ & $4-31$ \\
\hline
\end{tabular}

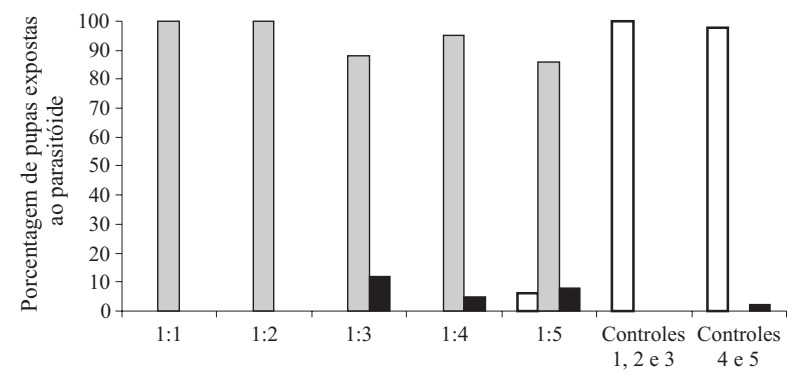

$\square$ Emergência de dípteros $\square$ Emergência de parasitóides $\square$ Pupas inviáveis

Fig. 3. Taxa de parasitismo em pupas de Cochliomyia macellaria expostas por 72 horas a fêmeas nulíparas de Nasonia vitripennis, utilizando-se diferentes relações de fêmeas parasitóides/hospedeiro.

Os resultados mostraram que o aumento na duração do desenvolvimento de $N$. vitripennis, quando exposta a um número crescente de hospedeiros, se deve às baixas densidades do parasitóide por pupa, reduzindo as condições micro-ambientais favoráveis ao seu desenvolvimento. Portanto, sugere-se que baixas densidades de parasitóides gregários por pupa hospedeira podem comprometer o desenvolvimento de parte, ou de toda a prole dos parasitóides (Tab. I). Podem, também, induzir a inviabilidade do hospedeiro, não havendo emergência de parasitóides, nem de hospedeiro (Fig. 3), comportamento verificado em densidades de parasitóide: hospedeiro acima de 1:2.

Aguiar-Coelho \& Milward-De-Azevedo (1996) estudaram o efeito da densidade de larvas de dípteros gregários, necrófagos e necrobiontófagos, incluindo $C$. macellaria, e observaram que, tanto as altas densidades de larvas de $C$. macellaria na dieta, quanto às baixas, mostraram-se deletérias para o desenvolvimento pósembrionário dos dípteros. No presente estudo, observaram-se também os efeitos deletérios em baixas densidades de parasitóide por pupa hospedeira, representados pelo aumento do desenvolvimento pósembrionário, uma menor emergência de parasitóides por pupa e uma menor taxa de parasitismo. Segundo SLANSKY \& SCRIBER (1985), o melhor desempenho de insetos gregários é obtido em uma faixa de densidade particular, ocorrendo declínio acima ou abaixo deste intervalo. Isso pode ocorrer em decorrência da temperatura alcançada por vários indivíduos numa mesma pupa. TURNER \& HowARD (1992), por exemplo, apresentaram a importância do calor metabólico no desenvolvimento pós-embrionário de alguns insetos, podendo existir diferenças que vão de cinco até $20^{\circ} \mathrm{C}$ de uma área colonizada pelos seus imaturos para uma não ocupada.

Por outro lado, não podemos deixar de destacar a discussão apresentada por CARDoso \& MilwARD-DEAzEvedo (1995), trabalhando com C. megacephala, para explicar a taxa de mortalidade das pupas em densidades elevadas de hospedeiro. Os autores consideraram como justificativa para esta observação o efeito deletério exploratório e alimentar das fêmeas de $N$. vitripennis, provocado pela ruptura do pupário do hospedeiro. Esta ruptura através do ovipositor pode ser realizada com a finalidade de permitir a ingestão de fluido do corpo do hospedeiro ou para permitir o reconhecimento das pupas já parasitadas, apresentando-se como uma estratégia da espécie para evitar o superparasitismo.

$\mathrm{O}$ fato de os machos terem sido mais influenciados do que as fêmeas, nas diferentes densidades, apresentando um aumento significativo na duração do desenvolvimento pós-embrionário, em relação à densidade do hospedeiro pode ser devida à maior sensibilidade às mudanças ambientais do microhabitat hospedeiro. Acredita-se que essa diferença entre os sexos se deva principalmente ao menor tamanho do macho, que influencia tanto no calor metabólico como nas suas necessidades nutricionais.

Em média emergiram 23,56 parasitóides por pupa hospedeira na densidade 1:1, havendo menor produtividade significativa quando o parasitóide foi exposto a mais de dois hospedeiros. Os resultados observados por CARDOSO \& MiLwARD-DE-AzEVEDo (1995) também mostraram uma queda significativa da produtividade por hospedeiro em função do aumento do número de hospedeiros, sendo a máxima encontrada em 1:1 e a mínima em 1:5, corroborando os dados obtidos no presente estudo. Observou-se que, em densidades acima de 1:2, parte das pupas muscóides produziram um pequeno número de parasitóides mostrando uma maior heterogeneidade na distribuição das posturas pelas fêmeas (ver o intervalo de variação na Tab. II). Estes resultados podem ser explicados por uma maior distribuição mais equiitativa das posturas pelas fêmeas parasitóides.

O gráfico de tendência exponencial mostrou que a proporção de fêmeas na prole aumenta em função do aumento de hospedeiros. Essa mesma tendência também foi observada por CARdoso \& Milward-DE-AzEvedo (1995). Muitas teorias vêm sendo apresentadas sobre a razão sexual em parasitóides. WyLIE (1966) sugere que o superparasitismo é um dos fatores diretamente responsáveis pelo aumento da porcentagem de machos na prole, corroborando nossos dados. Sugere-se que isto se deva a maior suscetibilidade dos machos às mudanças ambientais do microhabitat, talvez devido a seu tamanho diminuto em relação ao das fêmeas.

VAN DEN Asssem et al. (1984) apontaram que o tamanho do hospedeiro também pode influenciar na razão sexual de vespas parasitóides, como, onde os mais robustos apresentam uma tendência a um maior número de fêmeas na prole como verificado para Anisopteromalus calandrae (Howard, 1881) (Chalcidoidae, Pteromalidae). Porém o fator tamanho está fortemente relacionado com o fator densidade.

HARVEY \& Gols (1998) observaram uma variação da razão sexual de Muscidifurax raptorellus Kogan \& 
Legner, 1970 (Hymenoptera: Pteromalidae) em função do tamanho e da espécie do hospedeiro. Estes autores verificaram que proles oriundas de pupas pequenas de Musca domestica (Linnaeus, 1758) (Diptera, Muscidae) originaram um número maior de fêmeas, enquanto as pupas pequenas de Calliphora vomitoria (Linnaeus, 1758) (Diptera, Calliphoridae) apresentaram uma maior quantidade de machos. Os resultados obtidos para os pupários grandes foram o inverso dos resultados obtidos para os pequenos, sugerindo assim além do tamanho, uma possível influência da diferença nutricional interespecífica dos hospedeiros sobre a razão sexual dos parasitóides.

SHUKER et al. (2005) testaram uma hipótese chamada Asymetrical Local mate competition, que é a extensão da teoria de HamiLton (1967), Local mate competition $(L M C)$, que sustenta a tendência ao aumento da taxa de fêmeas na prole de espécies onde apenas as fêmeas abandonam o pupário dispersando-se. Porém, a versão SHUKER et al. (2005) trata de casos nos quais as fêmeas depositam seus ovos em diversos lugares, em tempos distintos, gerando emergências em tempo diferentes e encontros de machos competidores de ciclos diferentes. Neste teste, SHUKER et al. (2005) verificaram que as fêmeas de $N$. vitripennis possuem uma tendência a reduzir sua taxa de fêmeas na prole caso estejam utilizando um hospedeiro já parasitado ou caso haja um outro hospedeiro parasitado no mesmo local.

Logo pode-se concluir que o aumento do número de hospedeiros em relação aos parasitóides influenciaram na duração do desenvolvimento pós-embrionário, gerando um desenvolvimento mais lento de acordo com a redução da densidade dos parasitóides. O número da prole produzida por pupa hospedeira foi influenciado, havendo um declínio em função do número de hospedeiros por parasitóide. Já a razão sexual tendeu a um desvio para o nascimento de fêmeas com o aumento do número de hospedeiros. A taxa de parasitismo apresentou decréscimo quando o parasitóide foi exposto a mais de duas pupas hospedeiras.

Agradecimentos. À MSc. Ana Clara Gonçalves (Museu Nacional/UFRJ) pelo auxílio nas coletas; à Dra. Maria Angélica Penteado Dias (Departamento de Ecologia e Biologia Evolutiva da Universidade de São Carlos) pela ajuda na identificação dos parasitóides e à Fundação Rio-Zoo por ter permitido a realização de coletas, em especial ao Biólogo Anderson e ao Sr. Quirino.

\section{REFERÊNCIAS BIBLIOGRÁFICAS}

Aguiar-Coelho, V. M. \& Milward-de-Azevedo, E. M. V. 1996. Associação entre larvas de Cochliomyia macellaria (Fabricius) e Chrysomya albiceps (Wiedemann), Chrysomya megacephala (Fabricius) e Cochliomyia macellaria (Fabricius) (Calliphoridae, Diptera) sob condições de laboratório. Revista Brasileira de Entomologia 41(1):35-40.

Baumgartner, D. L. \& Greenberg, B. 1984. The genus Chrysomya (Diptera: Calliphoridae) in the New World. Journal of Medical Entomology 21:105-113.

1985. Distribution and medical ecology of the blow flies (Diptera: Calliphoridae) of Peru. Annual Entomological Society of America 78(5):565-587.
Cardoso, D. \& Milward-De-Azevedo, E. M. V. 1995. Influência da densidade de Chrysomya megacephala (Fabricius) (Diptera: Calliphoridae) sobre a capacidade reprodutiva de fêmeas nulíparas de Nasonia vitripennis (Walker) (Hymenoptera: Pteromalidae). Revista Brasileira de Entomologia 39(4):779-786.

Cardoso, D. \& Milward-De-Azevedo, E. M. V. 1996. Aspectos da biologia de Nasonia vitripennis (Walker) (Hymenoptera: Pteromalidae) em pupas de Chrysomya megacephala (Fabricius) e Chrysomya albiceps (Wiedemann) (Diptera: Calliphoridae), sob condições de laboratório. Revista Brasileira de Entomologia 40(2):143-146.

Cunha-E-Silva, S. L. \& Milward-De-Azevedo, E. M. V. 1994. Estudo comparado do desenvolvimento pós-embrionário de Cochliomyia macellaria (Fabricius) (Diptera, Calliphoridae) em duas dietas à base de carne, em laboratório. Revista Brasileira de Zoologia 11(4):659-668.

Ferreira, M. G. M. 1983. Sinantropia de Calliphoridae (Diptera) em Goiânia, Goiás. Revista Brasileira de Biologia 43(2):199-210.

Guimarães, J. H; Papavero, N. \& Prado, A. P. 1983. As miíases na região neotropical. Revista Brasileira de Zoologia 1(4):239-416.

Gulias-Gomes, C. C.; Soares, C. O. \& Milward-de-Azevedo, E. M V. 2003. Pupas de Chrysomya megacephala (Fabricius, 1794) (Diptera, Calliphoridae) crioconservadas em soluções de glicerol e dimetil sulfóxido como substrato de criação de Nasonia vitripennis (Walker, 1836) (Hymenoptera, Pteromalidae). Revista Brasileira de Zoociências 5(1): 101-120.

Hamilton, W. D. 1967. Extraordinary sex ratios. Science 156:477-488

Harvey, J. A. \& Gols, G. J. Z. 1998. The influence of host quality on progeny and sex allocation in the pupal ectoparasitoid, Muscidifurax raptorellus (Hymenoptera: Pteromalidae). Bulletin of Entomological Research 88:299-304.

Magalhães, M. N. \& Lima, A. C. P. 2001. Noções de Probabilidade e Estatística. IME-USP. 3ed. São Paulo $392 \mathrm{p}$.

Mello, R. P. 2003. Chave para identificação das formas adultas das espécies da família Calliphoridae (Diptera, Brachycera, Cyclorrhapha) encontradas no Brasil. Entomología y Vectores 10(2):255-268.

Moya-Borja, G. E. 2003. Erradicação ou manejo integrado das miíases neotropicais das Américas. Pesquisa Veterinária Brasileira 23:131-138

Paes, M. J. \& Milward-De-Azevedo E. M. V. 1998. Desenvolvimento pós-embrionário de Cochliomyia macellaria (Fabricius) (Diptera: Calliphoridae) criada em dietas naturais processadas em condições controladas. Parasitología al Dia 22:90-96.

Rueda, L. M. \& Axtell, R. C. 1985. Guide to common species of pupal parasites (Hymenoptera: Pteromalidae) of the house fly and other muscoid flies associated with poultry and livestock manure. North Carolina Agriculture Research Service Technical Bulletin, 278p.

SCHImidT, C. D. 1986. Nasonia vitripennis (Walker) a parasitoid contaminant in fly-rearing facilities. Southwestern Entomology 11(2):113-118.

Shuker, D. M.; Pen I.; Duncan A. B.; Reece S. E. \& West A. S. 2005. Sex Ratios under Asymetrical Local Mate Competition: Theory and a Test with Parasitoid Wasps. The American Naturalist 166(3):301-316.

Slansky, J. R. F. \& Scriber M. 1985. Food consumption and utilization. In. Compreensive Insect Physiology, Biochemistry and Pharmacology. Oxford, Pergamon, 162p.

Turner, B. \& Howard, T. 1992. Metabolic heat generation in dipteran larval agregations: a consideration for forensic entomology. Medical and Veterinary Entomology 6:179-181.

Wylie, H. G. 1966. Survival and reproduction of Nasonia vitripennis (Walker) on different host population densities. The Canadian Entomologist 98:273-286. 